(C) 2006 IEEE. Reprinted, with permission, from J. Hu and H. L. Tsai, "Droplet Acceleration in the Arc", IEEE International Conference on Industrial Electronics, Technology \& Automation (IETA06), Bridgeport, Connecticut, 2006.

This material is posted here with permission of the IEEE. Such permission of the IEEE does not in any way imply IEEE endorsement of any of the University of Bridgeport's products or services. Internal or personal use of this material is permitted. However, permission to reprint/republish this material for advertising or promotional purposes or for creating new collective works for resale or redistribution must be obtained from the IEEE by writing to pubs-permissions@ieee.org. By choosing to view this document, you agree to all provisions of the copyright laws protecting it. 


\section{DROPLET ACCELERATION IN THE ARC}

\author{
J. Hu \\ Department of Mechanical Engineering, \\ University of Bridgeport, \\ Bridgeport, CT 06604, USA
}

\author{
H.L. Tsai \\ Department of Mechanical and Aerospace Engineering, \\ University of Missouri-Rolla, \\ Rolla, MO 65409
}

\begin{abstract}
This paper simulates the acceleration of the droplet in the arc during gas metal arc welding process. After a droplet is detached from the electrode, it is accelerated in the high temperature and high velocity arc to the workpiece. The droplet is subjected to several forces, such as the arc plasma shear stress, arc pressure force, surface tension force, gravity force, and electromagnetic force. A comprehensive model is used to simulate the changes of droplet shape, temperature, and velocity during the acceleration in the arc. The transient interaction of droplet and arc plasma is through coupled boundary conditions, thus, no assumptions are needed to simulate the droplet acceleration. The simulated results were compared with the published experimental data and an agreement was found.
\end{abstract}

\section{INTRODUCTION}

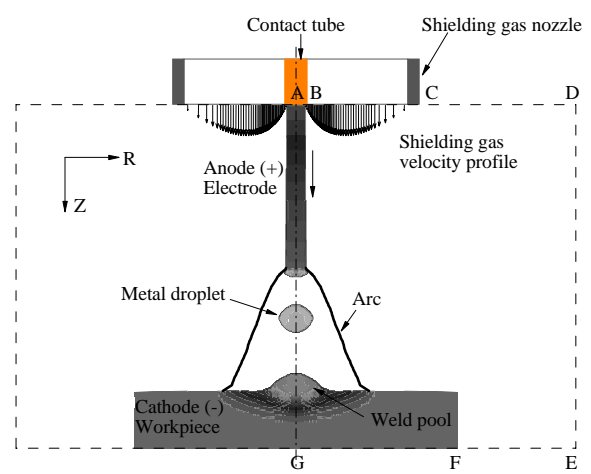

Fig. 1. A schematic representation of a GMAW system including the electrode, the arc, and the weld pool (not to scale).

Gas metal arc welding (GMAW) is an arc welding process that uses a plasma arc between a continuous, consumable filler-metal electrode and the weld pool, as shown in Fig. 1. The high temperature plasma arc melts the electrode and forms a droplet at the electrode tip. After a droplet is detached from the electrode, it is heated up and accelerated in the high temperature and high velocity arc. The accelerated high temperature droplet impinges onto the workpiece and a weld pool forms under the influences of the arc plasma and the periodical impingement of droplets. The influence of droplet impingement on the weld pool varies with the droplet temperature and size, the impingement frequency, and the impingement velocity. The transport of droplets into the weld pool is found largely responsible for the finger penetration commonly observed in the fusion zone [1]. A better understanding of the metal transfer process is important for improvements in the quality and productivity of welding.

Many researchers [2-8] have investigated the droplet impingement and weld pool dynamics. In their models, the droplet is often assumed to be spherical; the droplet size, temperature and impingement frequency are set to be a constant, the droplet acceleration is either not calculated [23] or calculated by applying a plasma drag force [4-7]. Tsao et al. directly assumed the droplet impingement velocity [2] in their model, thus, the droplet acceleration was not calculated. Fan and Kovacevic [3] calculated the droplet impingement velocity, but the droplet acceleration is not calculated, which was set to an experimentally measured value. Fan and Kovacevic [4,5] and Wang and Tsai [6,7] calculated the droplet acceleration by applying an arc plasma drag force on the droplet. As the arc model is not included, the acceleration of the droplet is calculated by applying an arc plasma drag force on the droplet.

The arc plasma drag force $F_{d}$ is calculated from an empirical formation [8] for a sphere immersed in a fluid stream of uniform velocity by

$$
F_{d}=\frac{\pi}{2} V_{e f f}^{2} \rho_{p} R_{d}^{2} C_{d}
$$

where $V_{\text {eff }}$ is the mean effective arc plasma axial velocity, which is taken as half of the arc plasma axial velocity, $\rho_{p}$ is the arc plasma density, $R_{d}$ is the droplet radius, $C_{d}$ is the drag coefficient. The drag coefficient $C_{d}$ depends on the Reynolds number and can be calculated from empirical formulas. Assuming the droplet have a spherical shape and is accelerated to the workpiece with a constant acceleration, the droplet acceleration due to the plasma drag force can be calculated as,

$$
a=\frac{3}{8} \frac{V_{e f f}^{2} \rho_{p} C_{d}}{R_{d} \rho_{m}}
$$

where $a$ is the acceleration of droplet due to plasma drag force, $\rho_{m}$ is the droplet density.

To calculate the plasma drag force exerted on the droplet using these formulations [4-8], it is required to assume the droplet has a spherical shape and is accelerated to the workpiece with a constant acceleration. The physical properties of the arc plasma, the drag coefficient, and the plasma velocity also have to be assumed. Given the range of temperature found in a welding arc, the appropriate value of the arc plasma properties is difficult to determine. There is also much uncertainty in the drag coefficient and the plasma 
flow velocity changes dramatically during the welding process. Furthermore, the shape of the droplet changes along the way as it is transported to the workpiece [9]. An effective method is needed to simulate droplet acceleration without assuming the droplet shape, the plasma properties, the drag coefficient, and the plasma velocity.

To accurately model the acceleration of droplet in the arc, the transient interaction of the droplet with the arc has to be considered. In this paper, a comprehensive model [10-11] is used to simulate the gas metal arc welding as an integrated system which includes both the metal domain and the arc domain. The electrode melting, droplet formation and transfer in the arc, droplet impingement onto the workpiece and weld pool dynamics are simulated in the metal domain. The heat transfer and fluid flow in the arc plasma and its surrounding gas environment are simulated in the arc domain. The transient interaction of the gas domain and metal domain are coupled through the energy, momentum, and current boundary conditions at each time step. The changes of droplet shape and temperature during droplet growth when it is still attached to the electrode and during the acceleration in the arc are modeled by simulating the fluid flow and heat transfer inside the droplet. The heat exchange between the arc and droplet and the momentum transfer from the arc to the droplet are obtained directly from the arc plasma calculation at each time step. Thus, no assumptions of the arc plasma temperature, properties and velocity and the drag coefficient, and droplet size and droplet shape are needed.

The direct and accurate measurement of droplet velocity and acceleration is difficult to conduct due to the complicated welding process of high speed and high temperature arc plasma. Jones et al. [12] have taken video images of droplets from the moment they were detached to the time they contacted the workpiece. Taken from the video images, the center positions of the droplets were then drawn with time as the flight trajectories. It was found that the flight trajectories could be fitted with quadratic curves within error limits. The first derivatives of these fitted curves were taken as the droplet velocities and the second derivatives were taken as the droplet accelerations. The simulated results are then compared with the published experimental data [12] and a reasonable agreement is found.

\section{MATHEMATICAL MODLELS}

Figure 1 is a schematic representation of a twodimensional axisymmetric GMAW system, with the computational domain marked by ABCDEFGA. There are three phases inside the computational domain: a solid phase, a liquid phase and a gas phase. The solid phase includes the unmelted electrode and part of the workpiece, while the liquid phase includes the melted electrode, falling droplet, and the weld pool on the workpiece. The gas phase includes the partially ionized arc plasma and shielding gas. Between the liquid zone and solid zone, there is a small zone called mushy zone where the solid and liquid metal coexist. A continuum formulation [13] was used to handle the metal domain consisting of the solid phase, liquid phase and mushy zone. Latent heat during melting and solidification was considered using the enthalpy method. As the properties of gas are far different from those of metal, two computational domains are used for computational robustness and efficiency. One computational domain is used to calculate the heat transfer and fluid flow in the gas phase and another is used for metal, which includes both solid phase and liquid phase. The heat transfer and fluid flow in both computational domains are coupled with the electromagnetic field.

The differential equations governing the conservation of mass, momentum, and energy based on the continuum formulation given by Chiang and Tsai [13] are employed in the present study, and the current continuity equation is used to calculate the current density distribution. The equations are given below:

Mass continuity

$$
\nabla \cdot(\rho \boldsymbol{V})=0
$$

Momentum

$$
\begin{aligned}
& \frac{\partial}{\partial t}(\rho u)+\nabla \cdot(\rho \mathbf{V} u)=\nabla \cdot\left(\mu_{l} \frac{\rho}{\rho_{l}} \nabla u\right)-\frac{\partial p}{\partial r}-\frac{\mu_{l}}{K} \frac{\rho}{\rho_{l}}\left(u-u_{s}\right) \\
& -\frac{C \rho^{2}}{K^{1 / 2} \rho_{l}}\left|u-u_{s}\right|\left(u-u_{s}\right)-\nabla \cdot\left(\rho f_{s} f_{l} \boldsymbol{V}_{r} u_{r}\right)-J_{z} \times B_{\theta} \\
& \frac{\partial}{\partial t}(\rho v)+\nabla \cdot(\rho \boldsymbol{V} v)=\nabla \cdot\left(\mu_{l} \frac{\rho}{\rho_{l}} \nabla v\right)-\frac{\partial p}{\partial z}-\frac{\mu_{l}}{K} \frac{\rho}{\rho_{l}}\left(v-v_{s}\right) \\
& -\frac{C \rho^{2}}{K^{1 / 2} \rho_{l}}\left|v-v_{s}\right|\left(v-v_{s}\right)-\nabla \cdot\left(\rho f_{s} f_{l} V_{r} v_{r}\right)+\rho g \beta_{T}\left(T-T_{0}\right)+J_{r} \times B_{\theta}
\end{aligned}
$$

Energy

$$
\begin{aligned}
& \frac{\partial}{\partial t}(\rho h)+\nabla \cdot(\rho \boldsymbol{V} h)=\nabla \cdot\left(\frac{k}{c_{s}} \nabla h\right)+\nabla \cdot\left(\frac{k}{c_{s}} \nabla\left(h_{s}-h\right)\right)-\nabla \cdot \\
& \left(\rho\left(\boldsymbol{V}-\boldsymbol{V}_{s}\right)\left(h_{l}-h\right)\right)-\Delta H \frac{\partial f_{l}}{\partial t}+\frac{J_{r}^{2}+J_{z}^{2}}{\sigma_{e}}-S_{R}+\frac{5 k_{b}}{e}\left(\frac{j_{r}}{c_{s}} \frac{\partial h}{\partial r}+\frac{j_{z}}{c_{s}} \frac{\partial h}{\partial z}\right)(6)
\end{aligned}
$$

Current continuity

$$
\nabla^{2} \phi=\frac{1}{r} \frac{\partial}{\partial r}\left(r \frac{\partial \phi}{\partial r}\right)+\frac{\partial^{2} \phi}{\partial z^{2}}=0
$$

Ohm's law

$$
J_{r}=-\sigma_{e} \frac{\partial \phi}{\partial r}, J_{z}=-\sigma_{e} \frac{\partial \phi}{\partial z}
$$

Maxwell's equation

$$
B_{\theta}=\frac{\mu_{0}}{r} \int_{0}^{r} J_{z} r d r
$$

In Eqs. (3)-(6), $u$ and $v$ are the velocities in the $r$ and $z$ directions, respectively. $\boldsymbol{V}_{\boldsymbol{r}}=\boldsymbol{V}_{\boldsymbol{I}}-\boldsymbol{V}_{\boldsymbol{s}}$ is the relative velocity vector between the liquid phase and the solid phase in the mushy zone. The subscripts $s$ and $l$ refer to the solid and liquid phases, respectively, and the subscript 0 represents the initial condition. $\quad p$ is the pressure; $T$ is the temperature; $h$ is the enthalpy; $\phi$ is the electrical potential; $\rho$ is the density; $\mu$ is the viscosity; $k$ is the thermal conductivity; $g$ is the gravitational acceleration; $\beta_{T}$ is the thermal expansion 
coefficient; $c$ is the specific heat; $\sigma_{e}$ is the electrical conductivity; $J_{r}$ and $J_{z}$ are current densities, in the respective $r$ and $z$ directions; $B_{\theta}$ is the self-induced electromagnetic field; $S_{R}$ is the radiation heat loss; $\mu_{0}$ is the magnetic permeability; $k_{b}$ is the Stefan-Boltzmann constant; and $e$ is the electronic charge.

The third and fourth terms on the right-hand side of Eqs. (4) and (5) represent the respective first- and second-order drag forces for the flow in the mushy zone. The fifth term on the right-hand side of Eqs. (4) and (5) represents an interaction between the solid and the liquid phases. The second term on the right-hand side of Eq. (6) represents the net Fourier diffusion flux. While the third term represents the energy flux associated with the relative phase motion, and the forth term is used to consider the latent heat of fusion. All the terms mentioned in this paragraph are zero, except in the mushy zone. When Eqs. (4)-(6) are used to calculate the arc plasma, these terms associated with the mushy zone are set to zero and all the thermal physical properties are replaced by those of the arc plasma.

The second-to-last term on the right-hand side of Eq. (5) is the thermal expansion term. The last term of Eq. (4) and Eq. (5) is the electromagnetic force term. The last three terms in Eq. (6) are Ohmic heating, radiation loss, and electron enthalpy flow, respectively.

The coupling of the metal domain and the arc domain are through boundary conditions. For the arc domain, the metal domain was treated as inner obstacles, while the arc plasma temperature, velocity, and pressure distributions were calculated. For the metal domain, a volume-of-fluid (VOF) method [13] was used to handle the free surfaces for the droplet and the surface of the weld pool. Additional body force source terms are added to the momentum transport equations at the metal free surface to consider the effects of surface tension, Marangoni shear stress, arc plasma shear stress and arc pressure. Additional source terms [10] are added to the energy equation for the special treatment of heat transfer near the anode sheath and the cathode sheath.

At the metal surface, surface tension pressure normal to the free surface can be expressed as [15]

$$
p_{s}=\gamma \kappa
$$

where $\gamma$ is the surface tension coefficient and $\kappa$ is the free surface curvature given by

$$
\kappa=-\left[\nabla \cdot\left(\frac{\vec{n}}{|\vec{n}|}\right)\right]=\frac{1}{|\vec{n}|}\left[\left(\frac{\vec{n}}{|\vec{n}|} \cdot \nabla\right)|\vec{n}|-(\nabla \cdot \vec{n})\right]
$$

where $\vec{n}$ is a vector normal to the local free surface which equals the gradient of the VOF function

$$
\vec{n}=\nabla F
$$

The temperature-dependent Marangoni shear stress at the free surface in a direction tangential to the local free surface is given by [4]

$$
\tau_{M s}=\frac{\partial \gamma}{\partial T} \frac{\partial T}{\partial \vec{s}}
$$

where $\vec{s}$ is a vector tangential to the local free surface.
The arc plasma shear stress is calculated at the free surface from the velocities of arc plasma cells immediately adjacent the metal cells

$$
\tau_{p s}=\mu \frac{\partial \boldsymbol{V}}{\partial \vec{s}}
$$

where $\mu$ is the viscosity of arc plasma.

The arc pressure at the metal surface is obtained from the computational result in the arc region. The surface forces are included by adding source terms to the momentum equations according to the CSF (continuum surface force) model $[15,16]$. Using $F$ of the VOF function as the characteristic function, the surface tension pressure, Marangoni shear stress, arc plasma shear stress, and arc pressure are all transformed to the localized body forces and added to the momentum transport equations as source terms at the boundary cells.

Only half of the entire physical domain is calculated due to the cylindrical symmetry along the centerline AG. The wire feed rate is incorporated through a boundary condition on axial velocity along AB. The imposed shielding gas flow is set through a boundary condition on axial velocity along BC. A constant mass flow boundary condition is used for the open boundaries $\mathrm{CD}$ and DE. The temperature boundaries along $\mathrm{AD}, \mathrm{DE}$, and $\mathrm{EG}$ are determined by the ambient condition, which is set as room temperature. Uniform current density is specified along AB. The voltage is set to zero at the bottom of the workpiece FG.

The current distribution is greatly influenced by the temperature in the arc column and the shape of the metal domain, but it is only slightly influenced by the temperature distribution in the metal domain as the electrical conductivity of metal varies slightly with temperature. Therefore, the current continuity equation and its associated boundary conditions are solved in the entire domain, while other primary variables, including $p, u, v$, and $T$, are calculated separately in the metal domain and the arc domain. The current continuity equation is iterated with the transport equations in the arc domain to obtain the current density distribution for both the arc domain and the metal domain. Iterations are required to assure convergence of each domain and then the boundary conditions are calculated from each domain for the coupling between the two domains.

For the metal domain, the method developed by Torrey et al. [14] was used to solve $p, u, v$, and $T$. This method is Eulerian and allows for an arbitrary number of segments of free surface with any reasonable shape. The basic procedure for advancing the solution through one time step, $\Delta t$, consists of three steps. First, at the beginning of the time step, explicit approximations to the momentum equations (4) and (5) are used to find provisional values of the new time velocities. Second, an iterative procedure is used to solve for the advanced time pressure and velocity fields that satisfy Eq. (3) to within a convergence criterion at the new time. Third, the energy equation is solved.

For the arc plasma domain, a fully implicit formulation is used for the time-dependent terms, and the combined convection/diffusion coefficients are evaluated using an 
upwind scheme. The SIMPLE algorithm [17] is applied to solve the momentum and continuity equations to obtain the velocity field. At each time step, the current continuity equation is solved first, based on the updated parameters. Current density and electromagnetic force are then calculated for the momentum and energy equations. The momentum equations and the continuity equation are then solved in the iteration process to obtain the new pressure and velocity. With the new pressure and velocity distributions, the energy equation is solved to get the new temperature distribution. Next, the temperature-dependent parameters are updated, and the program goes back to the first step to calculate the current continuity equation. This process is repeated for each time step until the convergence criteria are satisfied.

\section{RESULTS AND DisCUSSION}
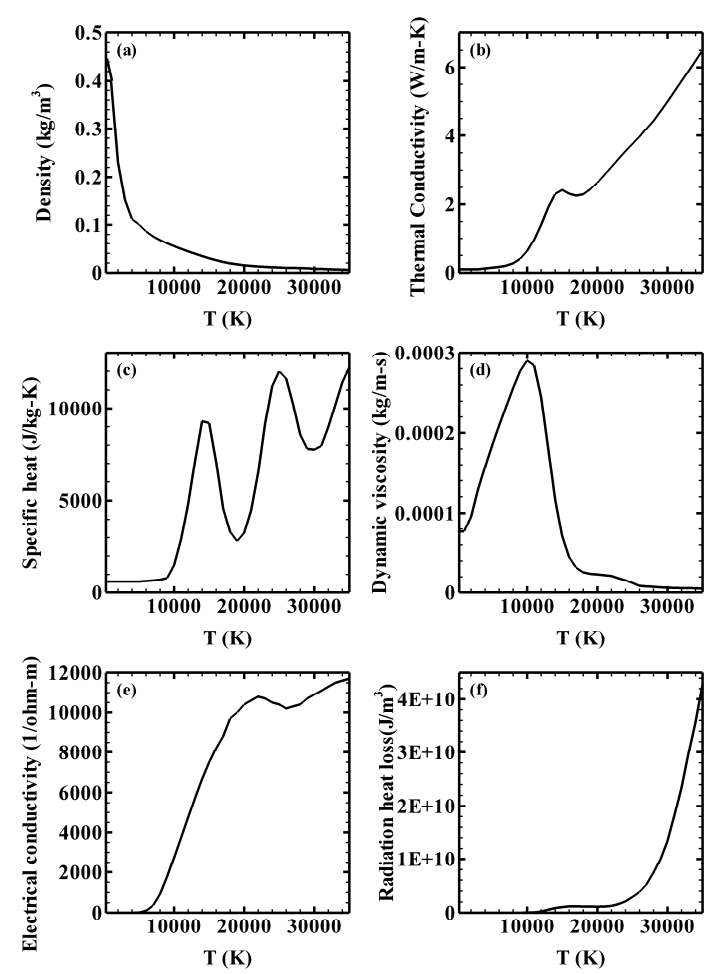

Fig. 2. Temperature-dependant material properties of argon and the volume radiation heat loss taken from [8].

The electrode is mild steel with a $0.16 \mathrm{~cm}$ diameter. The workpiece is also a mild steel disk with a $3 \mathrm{~cm}$ diameter and a $0.5 \mathrm{~cm}$ thickness. The shielding gas is argon and flows out of a $1.91 \mathrm{~cm}$ gas nozzle at a rate of $24 \mathrm{l} / \mathrm{min}$. The contact tube is set flush with the bottom of the gas nozzle and is 2.54 $\mathrm{cm}$ above the workpiece. The initial arc length is set as 0.8 $\mathrm{cm}$. Temperature-dependant material properties of argon and the volume radiation heat loss are taken from [8] and drawn in Fig. 2. The thermophysical properties of the solid and liquid mild steel are taken from [4] and listed in Table 1. Five current levels in the range of $200 \mathrm{~A}$ to $280 \mathrm{~A}$, with $20 \mathrm{~A}$ increases, are chosen to study the droplet acceleration at different current levels. For each of the five current levels, the temperature, velocity, arc pressure, and current density distributions are obtained by solving Eqs. (2)-(9), but only a full set of data is drawn for the current level of $220 \mathrm{~A}$ in Figs. 3-8.

Table 1. Thermophysical properties of mild steel and other parameters.

\begin{tabular}{lll}
\hline Nomenclature & Symbol & Value (unit) \\
\hline Specific heat of solid phase & $c_{s}$ & $700\left(\mathrm{~J} \mathrm{~kg}^{-1} \mathrm{~K}^{-1}\right)$ \\
Specific heat of liquid phase & $c_{l}$ & $780\left(\mathrm{~J} \mathrm{~kg}^{-1} \mathrm{~K}^{-1}\right)$ \\
Thermal conductivity of solid phase & $k_{s}$ & $22\left(\mathrm{~W} \mathrm{~m}^{-1} \mathrm{~K}^{-1}\right)$ \\
Thermal conductivity of liquid phase & $k_{l}$ & $22\left(\mathrm{~W} \mathrm{~m}^{-1} \mathrm{~K}^{-1}\right)$ \\
Density of solid phase & $\rho_{s}$ & $7200\left(\mathrm{~kg} \mathrm{~m}^{-3}\right)$ \\
Density of liquid phase & $\rho_{l}$ & $7200\left(\mathrm{~kg} \mathrm{~m}^{-3}\right)$ \\
Thermal expansion coefficient & $\beta_{T}$ & $4.95 \times 10^{-5}\left(\mathrm{~K}^{-1}\right)$ \\
Radiation emissivity & $\varepsilon$ & 0.4 \\
Dynamic viscosity & $\mu_{l}$ & $0.006\left(\mathrm{~kg} \mathrm{~m}^{-1} \mathrm{~s}^{-1}\right)$ \\
Latent heat of fusion & $H$ & $2.47 \times 10^{5}\left(\mathrm{~J} \mathrm{~kg}^{-1}\right)$ \\
Latent heat of vaporization & $H_{e v}$ & $7.34 \times 10^{6}\left(\mathrm{~J} \mathrm{~kg}^{-1}\right)$ \\
Solidus temperature & $T_{s}$ & $1750(\mathrm{~K})$ \\
Liquidus temperature & $T_{l}$ & $1800(\mathrm{~K})$ \\
Vaporization temperature & $T_{e v}$ & $3080(\mathrm{~K})$ \\
Ambient temperature & $T_{\infty}$ & $300(\mathrm{~K})$ \\
Surface tension coefficient & $\gamma$ & $\left.1.2(\mathrm{~N} \mathrm{~m})^{-1}\right)$ \\
Surface tension temperature gradient & $\partial \gamma / \partial T$ & $10^{-4}\left(\mathrm{~N} \mathrm{~m}{ }^{-1} \mathrm{~K}^{-1}\right)$ \\
Electrical conductivity & $\sigma_{e}$ & $7.7 \times 10^{5}\left(\Omega^{-1} \mathrm{~m}^{-1}\right)$ \\
Magnetic permeability & $\mu_{0}$ & $1.26 \times 10^{-6}\left(\mathrm{H} \mathrm{m}^{-1}\right)$ \\
Work function & $\phi_{w}$ & $4.3 \mathrm{~V}$ \\
Argon ionization energy & $V_{i}$ & $15.76(\mathrm{~V})$
\end{tabular}

Figures 3-8 show a sequence of the first droplet formation, detachment and transfer in the arc. The temperature and velocity distributions in the meal domain are shown in Figs. 3 and 4, respectively. The arc plasma temperature, velocity, and pressure distributions are shown in Figs. 5-7 and the current density distributions are drawn in Fig. 8.

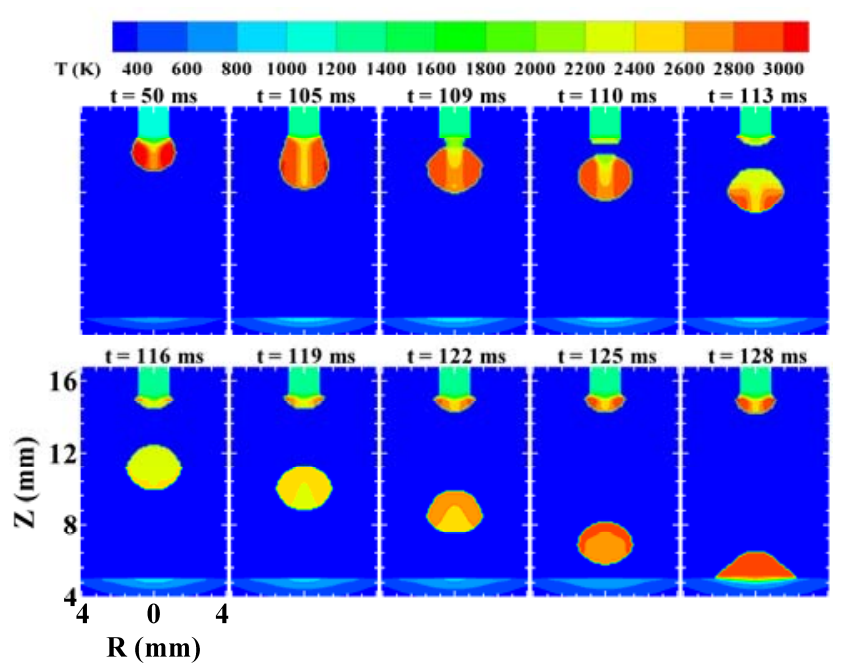

Fig. 3. Temperature distributions in the metal domain for $\mathrm{I}=220 \mathrm{~A}$.

After the droplet is detached, the temperature distribution in the droplet becomes more uniform through the mixing of fluid flow inside the droplet at the beginning of the separation. The detached droplet is then heated by the surrounding high temperature arc. The detached droplet is also subjected to the electromagnetic force, gravity, arc pressure, plasma shear stress, and surface tension. At the 
balance of these forces, the droplet is accelerated to the workpiece. These forces are also responsible for the changes of the droplet shape during the flight in the arc.

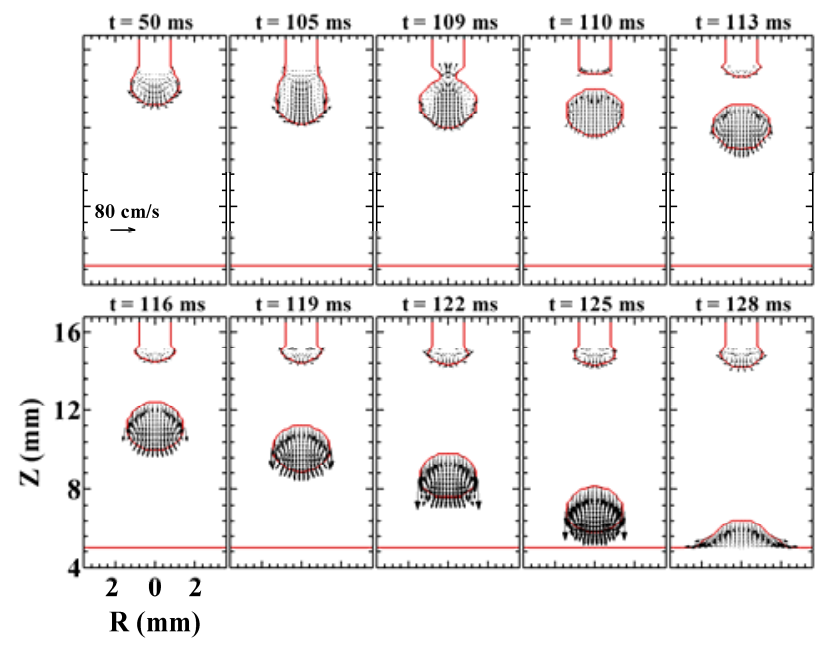

Fig. 4. Velocity distributions in the metal domain for $\mathrm{I}=220 \mathrm{~A}$.

$\mathbf{T}(\mathbf{K})$

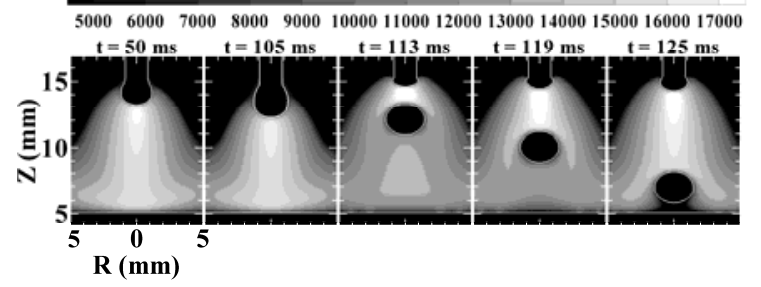

Fig. 5. Arc plasma temperature distributions for $\mathrm{I}=220 \mathrm{~A}$.

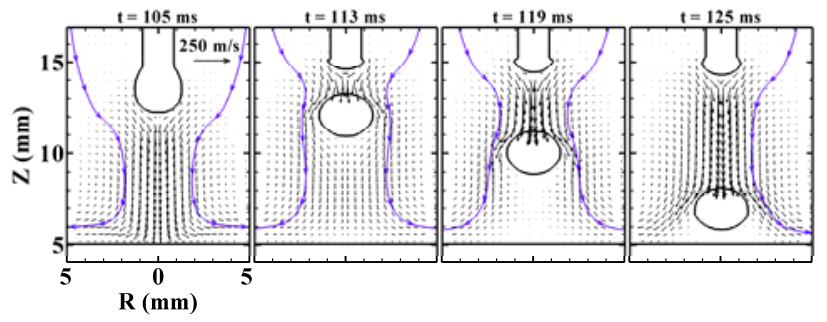

Fig. 6. Arc plasma velocity distributions for I $=220 \mathrm{~A}$.

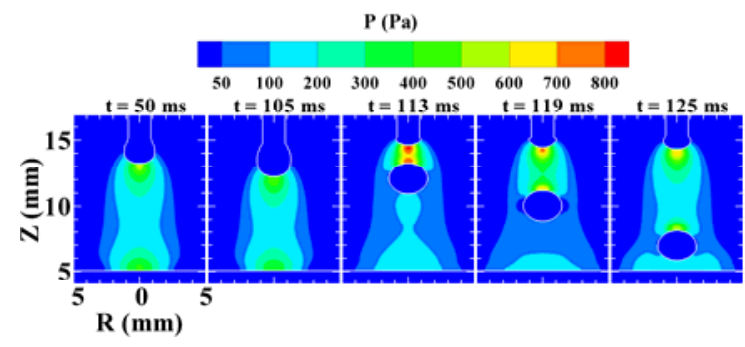

Fig. 7. Arc pressure distributions for $\mathrm{I}=220 \mathrm{~A}$.

As shown in Fig. 7, there are two high pressure regions before the droplet is detached. One is underneath the droplet, and the other is near the electrode. The high pressure underneath the droplet is caused by the pinch effect of the electromagnetic force, which draws arc plasma flow underneath the droplet. The pressure increase near the workpiece is due to the stagnation of the plasma flow impinging onto the workpiece. After the droplet is detached from the electrode, new arc plasma is struck between the electrode tip and the top surface of the detached droplet. There are two new high pressure regions, with one under the electrode tip and the other at the top surface of the droplet. The arc pressure difference between the top and bottom surfaces of the detached droplet propels the droplet down to the workpiece. The effect of the plasma shear stress is also remarkable in bringing down the detached droplet. From the plasma velocity distributions in Fig. 6, it can be seen that the plasma flow around the detached droplet is significant.

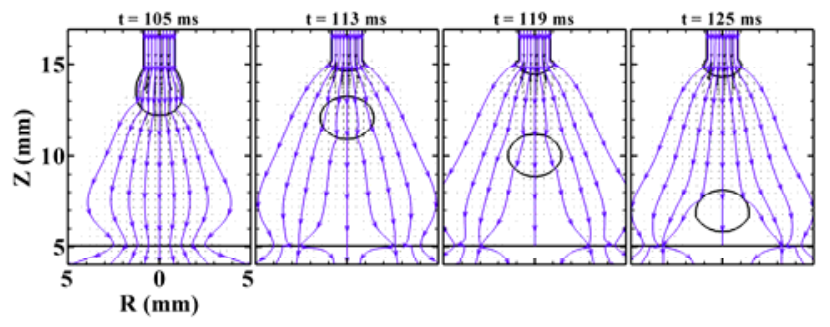

Fig. 8. Current density distributions for $\mathrm{I}=220 \mathrm{~A}$.
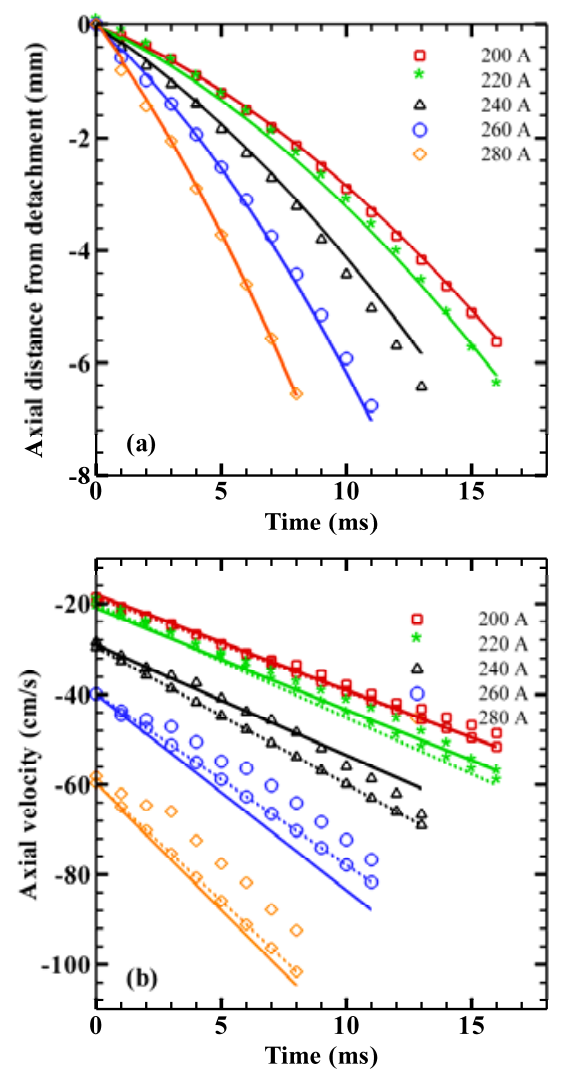

Fig. 9. Computational droplet positions and axial velocities compared with the experimental results at different currents. (a) Droplet flight trajectories; (b) Axial droplet velocities. In (a), the symbols are the droplet center positions from the computational results and the solid line is the fitted curve of the droplet trajectories from the experimental results of Jones et al. [12]. In (b), the symbols are the axial velocities at the droplet center from the computational results; the dashed lie is the axial velocities calculated by taking the first derivative of the computational trajectories; and the solid line is the velocities calculated by taking the first derivatives of the experimental trajectories. 
The arc pressure is high at the top surface of the detached droplet. The high arc pressure does not flatten the droplet, because of the effect of surface tension, which tries to maintain a round droplet shape. The oscillation of droplet shape from oblate to prolate is mainly the work of surface tension. However, the electromagnetic force also helps the droplet to resist being flattened to oblate by the arc pressure. From the current streamlines drawn in Figs. 8, it can be seen that current flows around the detached droplet. Except at the place where the droplet is close to the electrode tip, only a small amount of current flows through the detached droplet. When the droplet moves farther away from the electrode tip, less current flows through it and the electromagnetic force in the droplet also becomes smaller. As can be seen in Figs. 57 , the detached droplets have a more flattened shape near the workpiece than when they are near the electrode tip.

The droplet center positions from the computational results are compared with the fitted curves of the flight trajectories of Jones et al. [12] in Fig. 9(a). The solid lines are the fitted curves of the droplet trajectories from Jones et al. [12] and the symbols are the center positions of droplets from the computation. As can be seen, the calculated droplet center positions match the fitted curve well, except at some points near the electrode tip. The computational results show the droplets have a bigger acceleration at the early stage of the flight when they are near the electrode tip. While this bigger acceleration could not be shown in the fitted curve, as a constant acceleration was assumed, it was shown in the original flight trajectories made from the video images [12]. The bigger acceleration can also be explained by the computational results. From Figs. 6-7, it can be seen that the plasma flow between the electrode tip and the droplet is stronger and the arc pressure at the top surface of the droplet is higher when the distances between them are shorter. From the axial velocity distributions at the droplet center, which is drawn in Fig. 9(b) with only symbols, the trend of higher acceleration at the early stage is more obvious. The droplet velocities calculated by taking the first derivatives from both the fitted curves of the experimental and computational trajectories are drawn in Fig. 9(b). The droplet velocities calculated from the experimental trajectories are drawn with a solid line for each current level. The droplet velocities calculated from the computational trajectories are drawn with a dotted line and the symbol for each current level. The droplet velocities calculated from the trajectories match well for each current level. However, the velocities at the droplet center from the computational results do not fit well with them, especially at higher current levels. This is because the acceleration of the droplet is higher at the early stage. The droplet size, frequency and acceleration at different current levels are summarized in Table 2 with the corresponding experimental results.

\section{CONCLUSION}

A comprehensive model has been used to study the acceleration of the detached droplet in the arc during a gas metal arc welding process. After a droplet is detached from the electrode, it is further heated by the high temperature arc during the acceleration in the arc. The shape of the detached droplet changes in the arc at the balance of electromagnetic force, arc pressure, plasma shear stress, and surface tension. More flattened droplets were found near the workpiece than near the electrode tip, due to the smaller electromagnetic force acted on the droplet further away from the electrode tip. The detached droplet is accelerated to the workpiece by the pressure difference at its top and bottom surfaces and the plasma shear stress as plasma flows pass around the droplet. The acceleration of the detached droplets was higher at the early stage of the flight and was near constant at the later stage. The calculated droplet flight trajectory and droplet velocities are compared with the experimental results and a good agreement was found.

Table 2. Comparison of the droplet size, frequency, and acceleration at

\begin{tabular}{ccccc}
\hline $\begin{array}{c}\text { Current } \\
(\mathrm{A})\end{array}$ & $\begin{array}{c}\text { Wire } \\
\text { feed rate } \\
(\mathrm{cm} / \mathrm{s})\end{array}$ & $\begin{array}{c}\text { Droplet } \\
\text { radius } \\
(\mathrm{mm})\end{array}$ & $\begin{array}{c}\text { Droplet } \\
\text { frequency } \\
(\mathrm{Hz})\end{array}$ & $\begin{array}{c}\text { Droplet } \\
\text { acceleration } \\
\left(\mathrm{m} / \mathrm{s}^{2}\right)\end{array}$ \\
\hline 200 & $4.0(3.8)$ & $1.49(1.47)$ & $5.5(5.0)$ & $20.6(21.0)$ \\
220 & $4.5(4.3)$ & $1.32(1.34)$ & $9.2(8.8)$ & $25.0(22.4)$ \\
240 & $5.0(4.8)$ & $1.13(1.17)$ & $16.8(13.8)$ & $30.4(24.5)$ \\
260 & $5.5(5.3)$ & $0.95(0.97)$ & $31.5(23.8)$ & $38.0(43.8)$ \\
280 & $6.0(5.8)$ & $0.82(0.81)$ & $65.0(59.0)$ & $52.4(55.8)$ \\
\hline
\end{tabular}

Note: The experimental results shown in the parentheses are from Jones et al. [12]. The experiment was continuous constant current welding for 1.6 $\mathrm{mm}$ mild steel electrode shielded by $\mathrm{Ar}-2 \% \mathrm{O}_{2}$. The shielding gas flow rate was $24 \mathrm{l} / \mathrm{min}$ and the inner diameter of the nozzle was $19.1 \mathrm{~mm}$. The contacted tube was mounted flush with the bottom of the gas nozzle and was $25.4 \mathrm{~mm}$ above the workpiece.

\section{REFERENCES}

[1] C.H. Kim, W. Zhang, and T. DebRoy, "Modeling of Temperature Field and Solidified Surface Profile during Gas-Metal Arc Fillet Welding,” J. Appl. Phys., 94, 2003, pp. 2667-2679.

[2] Z. Cao, Z. Yang and X.L. Chen., "Three-Dimensional Simulation of Transient GMA Weld Pool with Free Surface,” Welding J., 2004, pp. 169-176s.

[3] H.G. Fan, and R. Kovacevic, "Dynamic Analysis of Globular Metal Transfer in Gas Metal Arc Welding - a Comparison of Numerical and Experimental Results,” J. Phys. D: Appl. Phys. 31, 1998, pp. 2929-2941

[4] H.G. Fan, and R. Kovacevic, "Droplet Formation, Detachment, and Impingement on the Molten Pool in Gas Metal Arc Welding,” Metall. Trans. 30B, 1999, pp. 791-801

[5] H.G. Fan, and R. Kovacevic, "A Unified Model of Transport Phenomena in Gas Metal Arc Welding including Electrode, Arc Plasma and Molten Pool”, J. Phys. D: Appl. Phys. 37, 2004, pp. 2531-2544.

[6] Y. Wang and H.L. Tsai, "Impingement of Filler Droplets and Weld Pool Dynamics during Gas Metal Arc Welding Process,” Int. J. Heat and Mass Transfer 44, 2001, pp. 2067-2080.

[7] Y. Wang and H.L. Tsai, "Effects of Surface Active Elements on Weld Pool Fluid Flow and Weld Penetration in Gas Metal Arc Welding," Metall. Trans. 32B, 2001, pp. 501-515.

[8] J.F. Lancaster, The Physics of Welding, Oxford Pergamon, $2^{\text {nd }}$ Edition, 1986, pp. 265-267.

[9] S. Subramaniam, D.R. White, D.J. Scholl, and W.H. Weber, "In Situ Optical Measurement of Liquid Drop Surface Tension in Gas Metal Arc Welding,” J. Phys. D: Appl. Phys., 31, 1998, pp. 1963-1967.

[10] J. Hu and H.L. Tsai, "Heat and Mass Transfer in Gas Metal Arc Welding, Part I: the Arc", Int. J. Heat Mass Transfer, 2006, in press

[11] J. Hu and H.L. Tsai, "Heat and Mass Transfer in Gas Metal Arc Welding, Part II: the Metal", Int. J. Heat Mass Transfer, 2006, in press

[12] L.A. Jones, T.W. Eagar and J.H. Lang, "A Dynamic Model of Drops Detaching from a Gas Metal Arc Welding Electrode,” J. Phys. D: Appl. Phys. 31, 1998, pp. 107-123.

[13] K.C. Chiang and H.L Tsai, "Shrinkage induced fluid flow and domain change in twodimensional alloy solidification”, Int. J. Heat and Mass Transfer, 35, 1992, pp. 1763-1770.

[14] M..D. Torrey, L.D. Cloutman, R.C. Mjolsness, and C.W. Hirt, "NASA-VOF2D: A Computer Program for Incompressible Flows with Free Surfaces,” LA-10612-MS, Los Alamos National Laboratory, 1985.

[15] J.U. Brackbill, D.B. Kothe, and C. Zemach, "A Continuum Method for Modeling Surface Tension,” J. of Computational Physics, 100, 1992, pp. 335-354.

[16] A. Celic, and G.G. Zilliac, "Computational Study of Surface Tension and Wall Adhesion Effects on an Oil Film Flow underneath an Air Boundary Layer," Nasa Ames Research Center, 1997

[17] Patanka, S.V., "Numerical Heat Transfer and Fluid Flow", New York: McGraw-Hill, 1980. 